\title{
Profesores noveles de educación física: percepciones sobre su formación docente en base al desempeño en el sistema escolar Novel physical education teachers: perceptions on their teaching training based on performance in the school system \\ *Claudio Hinojosa Torres, **Macarena Hurtado Guerrero, *Paula Magnere Ávalos *Universidad de Playa Ancha (Chile), ** Colegio Sagrados Corazones Padres Franceses de Viña del Mar (Chile)
}

\begin{abstract}
Resumen. La formación docente como actividad académica universitaria debe asegurar que cada futuro profesor sea capaz de responder a los diferentes requerimientos que tiene en la actualidad el sistema escolar. Aquí se devela una dificultad ya que el ingreso del docente novel a sus primeros pasos laborales está configurado como una etapa llena de incertidumbre y tensiones que no le permiten tener un desempeño óptimo. En este estudio se buscó comprender los significados otorgados por profesores noveles a su formación profesional desde la experiencia del ejercicio profesional. Para poder abordar el problema de estudio se orientó una investigación bajo el Paradigma Interpretativo con el método de la Fenomenología. Se utilizó la técnica Entrevista Semiestructurada para recoger datos de 15 profesores noveles (6 damas y 9 varones) utilizando el Análisis de Contenido y el Análisis de Discurso para el proceso de análisis de los datos. Como evidencia se resaltan las dificultades que presentan en el ejercicio profesional docente referido en habilidades para resolver problemas emergentes y el escaso manejo de aspectos administrativos y técnicos que implica el trabajo docente en la escuela.
\end{abstract}

Palabras clave: Profesor novel, Educación Física, Sistema escolar, Formación docente.

\begin{abstract}
Teacher training as a university academic activity must ensure that each trained teacher is able to respond to the different requirements that the school system currently establishes. At this phase, issues occur since novice teachers' first steps in the work environment are described as a stage full of uncertainty and tensions, not allowing them to perform at optimal levels. In this study, we sought to understand the meanings given by novice teachers to their professional training from the experience of professional practice. In order to address the study problem, an investigation was carried out under the Interpretative Paradigm, employing the Phenomenology Method. Semi-structured Interview technique was used to collect data from 15 new teachers (6 women and 9 men) using Content Analysis and Speech Analysis for the data analysis process. As highlighted, the difficulties encountered while exercising the profession referred to skills to solve emerging problems and the poor management of administrative and technical aspects involved in the school teaching work.
\end{abstract}

Key words: Novice Teacher, Physical Education, School System, Teacher Training.

\section{Introducción}

En Chile durante la primera parte de la década de los noventa se observó claramente la debilidad que traía el sistema de formación de docentes a raíz de los efectos de las reformas estructurales en educación durante la época militar, las que afectaron en su conjunto al sistema de educación superior, impactando especialmente en las carreras de pedagogía (Ávalos, 2002). Por otra parte, la profesión docente convivía con una constante desvalorización profesional durante esa época (Núñez, 2004), lo que significó la baja de la calidad en los programas de formación docente y la percepción negativa de los estudiantes sobre estos (Ávalos, 2009).

Con las reformas educativas de la década de los noventa, el rol de los docentes tomó mayor importancia debido a que la implementación de estas reformas tendría como actor principal a los profesores por ser un factor clave en la ejecución de diferentes tareas vinculadas a su ejercicio docente, entendiendo que la reforma educacional fue pensada e insertada desde el aula hacia la comunidad, lo anterior con la expectativa de generar un cambio sustantivo en la educación de escolares en el país.

A raíz de este escenario y teniendo claridad de las debilidades que presentaban los docentes en ejercicio profesional de aquel entonces, es que se implementó la política de Fortalecimiento de la Formación Inicial Docente (FFID), pues-

Fecha recepción: 31-10-19. Fecha de aceptación: 14-02-20

Claudio Hinojosa Torres

claudio.hinojosa@upla.cl ta en marcha el año 2000 en las 25 universidades (16 estatales y 9 privadas) que componen el Consejo de Rectores de Universidades en Chile (CRUCH), donde uno de sus principales ejes fue atraer estudiantes de excelencia a las carreras de pedagogía en base a diferentes tipos de becas, como también la incorporación de las prácticas pedagógicas como un mecanismo formativo para comprender e integrar los saberes teóricos y prácticos articulados en el contexto educacional y así dar respuesta a las diferentes necesidades que tendrá como futuro profesor al iniciar su carrera docente (Ávalos, 2003).

Este nuevo enfoque se sustentó en una sólida preparación académica basada en valores ético-morales y en el compromiso social, todo lo anterior basado en ámbitos como campos de formación que integran un conjunto de saberes que se articulan en torno a la formación de los estudiantes, existiendo configuraciones epistemológicas que diferencian los distintos contenidos por áreas de conocimiento y por los niveles de amplitud y metodologías con que se aborda su objeto, los cuales se plantean según Ávalos (2003) como: (a) Formación general: ámbito que aborda las principales líneas de pensamiento, enfoques y perspectivas que contribuyen a la comprensión de la realidad de los sujetos, de la realidad social y del conocimiento; (b) Formación profesional: ámbito que incluye el conjunto de saberes orientados a conocer, comprender y participar críticamente de los procesos de enseñanza y de aprendizaje que se desarrollan en los diferentes contextos y niveles educativos; (c) Formación de especialidad: ámbito que incluye saberes necesarios para la apropiación creativa del conocimiento de la disciplina correspon- 
diente al título; y (d) Formación práctica: ámbito que incluye los saberes y habilidades que se ponen en juego en el accionar del profesor, tanto en las aulas como en otras actividades que componen el ejercicio de su profesión.

El nuevo modelo de formación docente se fundamentó en la efectividad de los futuros docentes para transformar los modelos imperantes que establecían solo tareas de acumulación conocimiento teórico-conceptual propio de cada especialidad pedagógica, sino que además de eso, se vincule un aspecto de comprensión, interpretación y aplicación de la teoría en la realidad educativa y valorar las repercusiones culturales, sociales, personales y disciplinares de la educación en el país; esto significa lograr un proceso de formación integral permanente, lo cual permitirá al futuro docente saber actuar ante las necesidades y dificultades que le presente el ejercicio de la profesión (López, Pérez, Manrique \& Monjas, 2016; Paez \& Hurtado, 2019), estableciéndose con esto desafíos a las instituciones de educación superior por cuanto son las llamadas a entregar una formación integral que otorgue al profesor las habilidades que les permitan desempeñarse en contextos educativos diferentes (Urra, Fehrenberg, Muñoz, Matheu \& Reyno, 2019)

Con este nuevo escenario en Chile, las universidades (entre otros actores) apuntaron a fortalecer la formación de los futuros profesores en base al desarrollo de competencias pedagógicas y disciplinares necesarias para desempeñarse con altos estándares en el futuro ejercicio profesional (CPEIP, 2014), considerando las exigencias que significa el desempeño de profesores en los distintos espacios del sistema educativo, coincidiendo con Rodríguez, Del Valle \& De la Vega (2018) que expresan que los docentes requieren de competencias profesionales que les permitan enfrentarse al nuevo contexto educativo para que puedan llevar a cabo una educación de calidad.

De lo anterior se desprende que las universidades hayan centrado su atención en el estudiante desde todas sus dimensiones (Barrera \& Hinojosa, 2017), entendiendo que en el futuro profesional debe ser capaz de dar respuesta a una serie de requerimientos, como el ser proactivo, nutrirse de nuevos conocimientos, tener un sentido crítico y buscar soluciones a los problemas emergentes (entre otras características), que harán de él un profesor competente y comprometido con su labor, lo que para Díaz del Cueto \& Aguado (2012) ha significado un interés para profesionales e instituciones por determinar cuáles son las competencias que debe tener un docente para que se considere de calidad, entendiendo que para el logro de estos propósitos, la formación docente debe responder a la necesidad de poder entregar herramientas en todos los ámbitos que permitan al futuro docente leer la realidad socio-cultural para anticipar las necesidades de cambio y transformación social (López, Pérez, Manrique \& Monjas, 2016)

Es así como las exigencias antes descritas se vinculan innegablemente al inicio del trabajo de un docente y determinan, en gran medida, el éxito o el fracaso de esta primera etapa de integración a la vida profesional, complejizando las experiencias de aprendizaje que comenzaron con el proceso de formación universitaria y que tienen como cierre los primeros años de ejercicio profesional, sufriendo lo que Cornejo (1999) llama, a partir de Veenman (1984), como el «shock de la realidad» o también señalados como «shock de la transición» o «de la praxis» (Cornejo, 1999), en virtud de lo cual «los jóvenes profesores se ven enfrentados a una realidad que no corresponde a los esquemas ideales con los que les han formado» (Boerr, 2014), lo anterior en la lógica que los procesos de formación de las universidades no han articulados los modelos y teorías con la realidad imperante en el sistema educativo nacional, lo que según Boerr (2014) se puede considerar inclusive en el caso que la formación del estudiante haya sido excelente, en instituciones prestigiosas, con buenos profesores, con trabajo exigente e incluso con buenas calificaciones.

Es así como en el inicio de la carrera profesional, a los «nuevos docentes» o «docentes noveles» se les visualiza como «un profesor nuevo o novato que egresa de las aulas universitarias donde se formó con todo lo necesario para ejercer la profesión y con un proceso de práctica permanente en su tránsito formativo» (Vilca, 2005), el cual «se encuentra recientemente graduado, habitualmente joven, que se incorpora o inserta por primera vez en el ejercicio de la docencia, con un máximo de antigüedad en esta tarea de 3 o 5 años» (Alliaud, 2004; Cornejo, 1999; García, 2006; Gimeno \& Pérez, 2008; Imbernón, 1997). En cualquiera de los dos casos, este nuevo docente cuenta con las competencias necesarias para poder ejercer su profesión pero presenta la dificultad de no tener experiencia por su condición de novato, lo que condiciona sus acciones y lo dificulta en la toma de decisiones, convirtiéndose esta etapa en un periodo complejo, crítico y determinante para la consolidación de sus competencias pedagógicas y/o profesionales, las que les permitirían planear, organizar y ejecutar situaciones de enseñanza y aprendizaje en pro de la formación de los alumnos (AlmonacidFierro, Merellano-Navarro, Feu, Vizuete \& Orellana, 2019).

Por consiguiente, en esta etapa debe considerarse la orientación y acompañamiento para estos docentes puesto que, si las experiencias iniciales son gratificantes, la impresión y transferencias serán positivas, o en su defecto, si las primeras experiencias son negativas y van asociadas a sentimientos de desencanto y fracaso, la impresión será negativa y desarrollará comportamientos similares en el futuro. Es así como en muchos casos «un refuerzo continuado de experiencias negativas puede resultar en la decisión de finalizar la carrera docente» (Gold, 1996).

Asimismo, cuando el profesor novel se incorpora por primera vez a un centro escolar lo hace con la ilusión y preocupaciones lógicas de un novato, el cual al iniciar el ejercicio de su profesión siente la necesidad y anhelo de ser aceptado, de asimilar la forma de ser frente a los alumnos, de cómo salir de situaciones problemáticas y de aplicar lo aprendido durante su formación inicial, lo que puede resultar una compleja tarea según Bores et al. (2020) puesto que debe dilucidar qué métodos didácticos pueden adaptarse mejor a una determinada realidad educativa en centros escolares, sobre todo si no cuenta con la experiencia necesaria para realizar tales juicios.

En investigaciones realizadas en diversos países (Calvo \& Camargo, 2015; Carreño, Velasco, Domínguez \& Martín, 2019; Huberman, 1988 en Vilca, 2005; Vonk, 1996) se ha comprobado que la problemática de los profesores noveles no es igual a la de aquellos profesores con algunos años de 
experiencia en el ejercicio docente. Para un profesor que se incorpora al sistema educativo se inicia un periodo profesional de acomodación a su nueva labor, que según diversas circunstancias puede durar algunos años (Vilca, 2005), donde el docente centra su atención, según Carreño et al. (2019), en la sistematización de los procesos que le permiten la comprensión de las relaciones entre la teoría y la práctica.

En el caso específico de la formación inicial del profesor de Educación Física, se afirma que el aprendizaje de la formación docente no comienza con el programa inicial del curso ni termina con la obtención del título profesional, sino que es algo que el profesor realiza durante toda su vida (Carreiro da Costa, Carvalha, Diniz, \& Pestana, 2006). Al respecto, Carreiro da Costa et al. (1996) complementa afirmando que el estudiante de la carrera de Educación Física, en la mayoría de los programas de formación inicial debe adquirir los conocimientos científicos, pedagógicos y las competencias necesarias para enfrentar adecuadamente la carrera docente, lo que para Almonacid-Fierro et al. (2019) no siempre se cumple ya que «el vínculo entre la formación inicial docente y la realidad de la escuela genera en la mayoría de las veces un cierto divorcio, que se acentúa en los déficits de carácter pedagógico con los que llegan los profesores al ejercicio de la docencia», manifestándose en el débil dominio de las aulas y su diversidad, además de no tener estrategias para enfrentar las condiciones ideales de infraestructura e implementación especifica de la asignatura.

A la luz de lo expuesto, el docente novel pretende conseguir un acercamiento entre el alumnado y la materia que imparte, siendo éste consciente de que el verdadero compromiso por el aprendizaje parte de un interés personal, no de una obligación impuesta de fuera (Cañadas, Santos-Pastor \& Castejón, 2019; Fernández, 2005). De esta forma el docente de educación física tiende a propiciar una comunicación abierta y transversal, en función de la realidad con que el aula se le presenta (patio, gimnasio, cancha, etc.), motivo por el cual requiere de herramientas para formar un clima y ambiente que propicie realizar las clases en condiciones favorables para el aprendizaje de los alumnos, lo que según Cañadas et al. (2019) supone que el docente deberá diseñar las tareas vinculadas al contenido y poner en práctica con actividades según diferentes técnicas, estilos y estrategias para la enseñanza, considerando la evaluación como cierre del proceso de enseñanza y aprendizaje; es por lo anterior que las competencias asociadas a la práctica favorecen las respuestas a estos requerimientos y posibilita determinar acciones para responder a las situaciones que se presentan, siendo parte de esto los procesos de auto reflexión como retroalimentación de su desempeño.

En la actualidad y en conformidad con las exigencias que se han integrado al sistema educativo chileno, es que la formación de profesores se ha fortalecido a través del aumento de las exigencias de ingreso para quienes se formen como profesores (como lo mandata la Ley 20.903), la organización de los procesos de formación a través de estándares orientadores para carreras de pedagogía en educación física (CPEIP, 2014), lo anterior como mecanismo para asegurar la formación docente acorde a los requerimientos actuales, y disponiendo de un sistema de aseguramiento de la calidad en base a un sistema de acreditación obligatoria para las carreras de pedagogía, lo que se refuerza con la Ley 20.903: Sistema de Desarrollo Profesional Docente, que dentro de sus apartados dispone una normalización a la carrera docente, impactando con la integración de altos estándares de exigencia a las instituciones de educación superior que opten por formar profesores.

\section{Material y método}

El presente estudio se basa en los principios del Paradigma Interpretativo el cual permite un acercamiento profundo a la realidad, entendiendo que no existe una sola verdad sino que emerge de los distintos significados que le dan las personas a las situaciones en que se encuentran (Briones, 1999). Esta orientación metodológica asume una concepción de los fenómenos sociales como construcciones de significado hechas a través del lenguaje, por lo que la realidad no sería un objeto externo ni estático, sino una construcción social de percepciones y significados (Delgado \& Gutiérrez, 1995; Vasilachis de Gialdino, 2006). Se enmarca como Fenomenológica ya que este tipo de investigación se centra en como las personas comprenden los significados de los eventos a partir de la experiencia subjetiva y con ello se descubren las formas de comprensión que la gente tiene de fenómenos específicos para encuadrarlos dentro de categorías conceptuales (Ortiz, 2015). Su alcance temporal es de tipo transversal dado que se recolectaron los datos en un solo momento, en un tiempo único (Liu, 2008 y Tucker, 2004 en Hernández, Fernández \& Baptista, 2014).

Como técnica de recolección de información se utilizó la entrevista semiestructurada (Corbetta, 2007; Valles, 2002), la cual se funda en la necesidad de conocer sin interferir ni manipular la realidad objeto y ayudar al recuerdo preciso de los eventos para poco a poco se reconstruir un recuerdo (Keats, 2009) e ir entrelazando temas para ir construyendo un conocimiento holístico y comprensivo de la realidad (Bizquerra, 2009), para lo cual el entrevistador adopta el formato de estímulo-respuesta sin esperar la respuesta objetivamente verdadera, sino subjetivamente sincera (Denzin \& Lincoln, 2015; Ruiz, 2012).

En relación al instrumento, este se conformó por un guion de ocho preguntas abiertas no dicotómicas, las cuales buscaron explorar profundamente el fenómeno de estudio propiciando que emergieran factores y asuntos referidos a este, dándole plena libertad de respuesta al entrevistado (Keats, 2009). Las preguntas fueron: (a) ¿Qué asignaturas en tu formación fueron significativas para el desempeño en el sistema escolar?; (b) ¿Qué importancia tuvieron las prácticas para tu desempeño actual como profesor en el sistema escolar?; (c) ¿Qué experiencias de tu formación docente fortalecieron tus aspectos profesionales para el desempeño en el sistema escolar?; (d) ¿Qué apoyos has recibido en esta etapa inicial de tu desempeño profesional?; (e) ¿Cuáles son las mayores dificultades que has enfrentado al iniciar el recorrido como profesor?; (f) ¿Qué herramientas recibidas durante tu formación han ayudado a resolver las dificultades presentes en tu profesión?; (g)¿Qué idea de la realidad profesional lograste dimensionar durante la formación como docente?; y (h) ¿Los primeros años de ejercicio profesional logran satisfacer tus expectativas como docente?. Este grupo de preguntas se 
sometió a un juicio valorativo por parte de 3 expertos, los cuales a través de un cuestionario de validación entregaron una valoración de 1 a 4 a cada pregunta, entendiendo que el nivel 4 era el que consideraba un alto nivel de propiedad y coherencia entre la pregunta, los objetivos y el fenómeno de estudio.

En cuanto a la muestra, esta fue de tipo intencionada según determinados criterios de inclusión, que para Izcara (2014) se conforma por «criterios establecidos por el investigador y con esto se seleccionan los casos que cumplen con uno o varios criterios específicos», además de integrar a la muestra casos ricos en información para el estudio en profundidad (Patton, 1990). De esta forma el estudio se compuso de una muestra de profesores y profesoras de Educación Física titulados de 4 universidades (dos públicas y dos privadas) de la $\mathrm{V}^{\mathrm{a}}$ región de Valparaíso, Chile, considerando como criterios de inclusión: (a) Docentes de 1 a 5 años de experiencia en el sistema escolar; (b) Cumplir carga horaria mínima de 22 horas pedagógicas semanales; y (c) Que los establecimientos educacionales donde ejercían se encontraran en la $V^{\mathrm{a}}$ región de Valparaíso. Con los criterios declarados se fundamenta la búsqueda de aquellos informantes más idóneos y representativos de la población de estudio por su conocimiento de la situación o del problema a investigar (Ruiz, 2012). Es así como la muestra fue conformada en total por quince profesores (seis damas y nueve varones).

Los docentes que cumplían con los requisitos fueron contactados personalmente y voluntariamente aceptaron ser parte de la investigación, para en una segunda etapa generar el acercamiento y desarrollar las entrevistas, no sin antes solicitarles firmar un carta de consentimiento informado donde se les daba a conocer los alcances del estudio y solicitarles la autorización para el uso de los datos entregados.

Como estrategia de análisis de datos se utilizó en un primer momento el Análisis de Contenido (AC), procedimiento se reduce al tratamiento estadístico de diferentes unidades de registro (palabras, temas, etc.), es decir, al conteo del número de veces que se repite cada unidad de registro (Izcara, 2014), siendo una técnica laboriosa que requiere de disciplina, perseverancia y rigor, además de intuición, para captar aquellos elementos más relevantes (Grawitz, 1984; Pérez, 2002). Este primer paso debe permitir un elevado grado de flexibilidad entendiendo que este primer análisis se queda en la superficie y el investigador debe considerar que las palabras no tienen significados unívocos y descontextualizados, por el contrario, el significado depende del contexto en que se encuentran insertas (Grawitz, 1984; Denzin \& Lincoln, 2015).

La segunda parte del tratamiento de datos se desarrolló de acuerdo a la técnica de análisis de discurso (AD) la cual «estudia el orden, el desorden y la organización de la acción social cotidiana, aprehendiendo lo que la gente dice, cuenta o hace $y$, en definitiva, todo aquello tal y como es producido por los participantes en la conversación» (Artiles, 1990; Dooley \& Levinsohn, 2007; Iñiguez, 2011). Con este procedimiento, el investigador al analizar la producción de datos es capaz de identificar, describir y estudiar el orden que se produce, abriéndose a develar las evidencias necesarias para el proceso de comprensión del fenómeno de estudio, interactuando subjetivamente entre lo dicho, lo comprendi- do y la teoría existente (Barrera \& Hinojosa, 2017).

El procedimiento de análisis se realizó con el software Atlas.ti 7, que clasifica en la categoría de los «constructores de teorías basadas en códigos» (Flick, 2007), el cual permite organizar y sistematizar la información para desarrollar un análisis reflexivo y en diálogo con los datos, posicionando al investigador en una lógica inductiva y naturalista para lograr la mayor apertura y sensibilidad posible a los datos, revelando patrones, tendencias, características y otras dimensiones de interés en la investigación. De esta manera, la obtención de un resultado pasa por dos momentos: (a) Decodificación de los datos obtenidos por el investigador que emanan de las creencias y los significados que para los sujetos tienen los hechos relacionados con el fenómeno de estudio llamada también «perspectiva emic»; y (b) Recodificación de la información que lleva la impronta teórica del investigador conocida como «perspectiva etic» (Álvarez, 2007).

\section{Análisis y resultados}

Las diversas posibilidades para presentar los resultados en investigación cualitativa se pueden ubicar en dos ejes: en un extremo está el propósito de desarrollar una teoría a partir de los datos y las interpretaciones según el modelo de Strauss (1987) y Strauss \& Corbin (2002), y según Van Maanen (2011) en el otro extremo están los relatos desde el campo que intentan ilustrar las relaciones que el investigador busca fundamentar.

De esta forma y siguiendo un orden la presentación de resultados, se desarrolló en primer lugar análisis de contenido, el cual determinó un primer acercamiento al conjunto de datos y, en primer lugar, dio muestra de una serie de palabras o conceptos que constituían gran parte de los discursos que componían las respuestas de los sujetos, las cuales se organizadas desde la que tenía mayor a la de menor frecuencia, entendiendo que este primer paso solo constituye un ejercicio de conteo de palabras y le entrega al investigador una orientación general de temas que podrían estar siendo tratados en él cuerpo general de datos, lo que puede verse reflejado en la tabla 1.

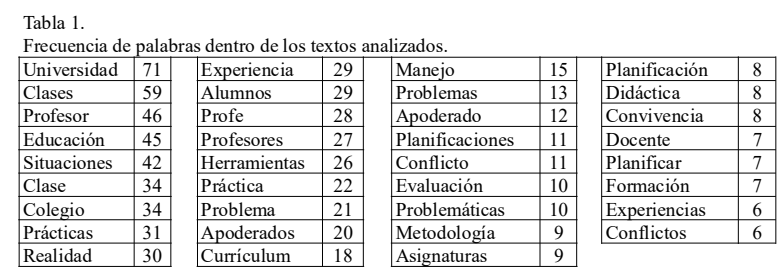

Los conceptos que se desprenden del AC se pueden vincular entre sí por su significado, generándose acercamientos semánticos entre ellos, lo que determina como resultado los Conglomerados Temáticos (11 en total), que pueden entenderse como «grupos de palabras en función de su similitud de aparición o significado en los diferentes archivos, nodos o casos» (Trigueros, Rivera \& Rivera, 2018), los que contienen conceptos en función de su relación semántica, lo que se refleja en la tabla 2.

Este segundo paso de presentación de resultados establece el punto de partida para el trabajo del Análisis de Discurso en la investigación, develando el primer acercamiento 


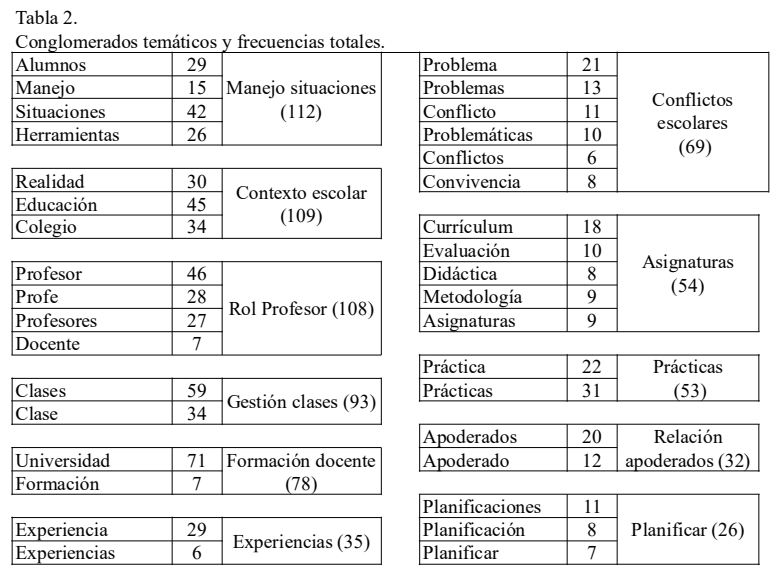

a los temas que están siendo tratados por los textos. Esto se condice con la posibilidad de observar preliminarmente los temas orientadores de los textos, entendiendo que el siguiente nivel de análisis deberá intencionar la estructura y significado a través de oraciones y frases, e intentar a través de la deconstrucción del texto reconstruir codificaciones en categorías o familias de códigos.

En una tercera etapa se desarrolló un proceso inductivointerpretativo orientado por el análisis del discurso, el cual buscó y seleccionó unidades de registro o citas, entendidas como «la unidad básica de análisis, como una primera selección del material de base, una primera reducción de la información» (Patton, 1990). En esta misma lógica Muñoz y Sagahún (2017) señalan que las citas pueden ser fragmentos de texto (palabras, frases, enunciados, párrafos), secciones rectangulares dentro de una imagen, lapsos en un archivo de audio o video, o puntos en un mapa. De lo anterior se desprende que de los 15 documentos primarios (entrevistas) surgen 156 citas o unidades de registro (ver tabla 3) que representan los fragmentos que el investigador rescata con énfasis en el significado otorgado por el sujeto en el discurso construido.

$$
\begin{aligned}
& \text { Tabla 3. Distribución total de citas por documento primario. } \\
& \begin{array}{|l|l|l|l|l|l|l|l|l|l|l|l|l|l|l|l|}
\text { D1 } & \text { D2 } & \text { D3 } & \text { D4 } & \text { D5 } & \text { D6 } & \text { D7 } & \text { D8 } & \text { D9 } & \text { D10 } & \text { D11 } & \text { D12 } & \text { D13 } & \text { D14 } & \text { D15 } & \text { Total } \\
\hline
\end{array}
\end{aligned}
$$

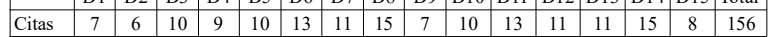

Si bien la distribución de la cantidad de citas por documento primario varía según cada sujeto, la distribución de ellas fue bastante homogénea, considerando que los sujetos manifiestan libremente sus opiniones sin una direccionalidad por parte del investigador. De esta forma este primer nivel del análisis de discurso con las citas capturadas en los textos da paso al trabajo de codificación o clasificación de citas en los códigos emergentes.

Con la etapa de citación concluida, comienza el proceso de codificación el cual puede estructurarse bajo dos orientaciones según Muñoz \& Sagahún (2017): a) Una deductiva, lo que implica partir de una teoría e intentar contrastar con los datos y dar cuenta de ella, predefiniendo códigos donde la tarea sería encontrar citas que se asocien a esos códigos; o b) Una inductiva, donde los códigos se fundan a partir de la lectura e interpretación de los datos en los cuales se identifican fragmentos significativos.

Entendiendo que, por la naturaleza de la investigación, la orientación inductiva logra mayor riqueza en el análisis, se dio paso al trabajo de agrupamiento por cercanía del significado o acercamiento semántico de las citas el cual busca englobar dentro de una misma entidad o concepto elementos (en este caso fragmentos de texto) que tienen un denominador común. Supone, por tanto, el primer paso dentro del proceso de abstracción dentro del trabajo cualitativo (Penalba, Alaminos, Francés \& Santacreu, 2015), por lo que un código puede entenderse como «una palabra o frase corta que simbólicamente asigna un atributo sumativo, saliente, capturador de la esencia, y/o evocativo a una porción de datos textuales o visuales» (Saldaña, 2009 en Muñoz \& Sagahún, 2017).

Con el término del proceso de codificación, se generan 15 códigos, los cuales contienen un número de citas que los conforman y que puede verse en la tabla 4.

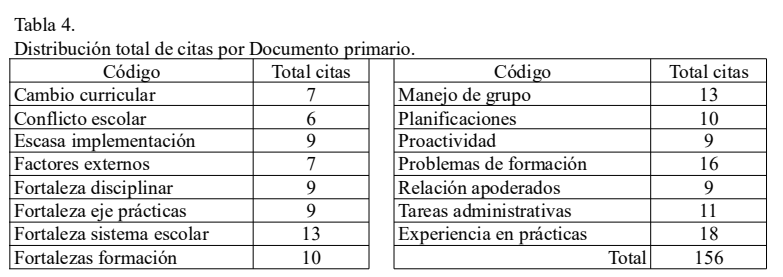

De esta manera se crearon 15 códigos, los cuales fueron definidos operacionalmente, que de acuerdo a Cazau (2006) esta definición se entiende «como una descripción que se construye, adapta o traduce a partir de las características observables del fenómeno (variables o unidades de análisis), incorporando los elementos concretos, empíricos o indicadores del hecho que se investiga o al tema o contenido representado», generándose las definiciones que se obser-

\begin{tabular}{|c|c|}
\hline Código & Definición operacional \\
\hline Cambio curricular (CC) & $\begin{array}{l}\text { Implica la preparación por parte de la universidad para enfrentar las } \\
\text { transformaciones de orden curricular en el sistema escolar. }\end{array}$ \\
\hline Conflicto escolar (CE) & $\begin{array}{l}\text { Dificultades que tiene el profesor para resolver las situaciones de } \\
\text { conflicto que se presentan diariamente en el sistema escolar. }\end{array}$ \\
\hline $\begin{array}{l}\text { Escasa implementación } \\
\text { (EI) }\end{array}$ & $\begin{array}{l}\text { Da cuenta de las limitaciones didácticas en la clase a raíz de la escasa } \\
\text { implementación de material didáctico. }\end{array}$ \\
\hline Factores externos (FE) & $\begin{array}{l}\text { Factores como la tecnología o el ambiente familiar y/o social de los } \\
\text { estudiantes que dificultan el ejercicio docente. }\end{array}$ \\
\hline $\begin{array}{l}\text { Fortaleza disciplinar } \\
\text { (FD) }\end{array}$ & $\begin{array}{l}\text { Conocimiento teórico-práctico de las áreas disciplinares que } \\
\text { componen la formación docente en un alto estándar de logro. }\end{array}$ \\
\hline $\begin{array}{l}\text { Fortaleza eje prácticas } \\
\text { (FEP) }\end{array}$ & $\begin{array}{l}\text { Impacto favorable en la formación a través de la participación de las } \\
\text { prácticas como articulador de la teoría y la praxis. }\end{array}$ \\
\hline $\begin{array}{l}\text { Fortalezas sistema } \\
\text { escolar (FSE) }\end{array}$ & $\begin{array}{l}\text { Aspectos favorables que presenta el sistema escolar para el buen } \\
\text { desempeño profesional de los docentes. }\end{array}$ \\
\hline $\begin{array}{l}\text { Fortalezas formación } \\
\text { (FF) }\end{array}$ & $\begin{array}{l}\text { Acciones en la formación que cumplen con competencias necesarias } \\
\text { para la consecución de un profesor de excelencia. }\end{array}$ \\
\hline Manejo de grupo (MG) & $\begin{array}{l}\text { Falta de experiencia del docente para tener manejo del grupo curso y } \\
\text { poder tener el dominio pedagógico de la clase. }\end{array}$ \\
\hline Planificaciones (PL) & $\begin{array}{l}\text { Alude a las habilidades del profesor para programar enseñanza a } \\
\text { través de sus planificaciones anuales, de unidad y de sesión. }\end{array}$ \\
\hline Proactividad (PR) & $\begin{array}{l}\text { Autoaprendizaje complementario de los estudiantes en los diferentes } \\
\text { ámbitos que integra la formación docente. }\end{array}$ \\
\hline $\begin{array}{l}\text { Problemas de } \\
\text { formación (PF) }\end{array}$ & $\begin{array}{l}\text { Indica de las debilidades que han enfrentado en el ejercicio } \\
\text { profesional y que provienen de su formación universitaria. }\end{array}$ \\
\hline $\begin{array}{l}\text { Relación apoderados } \\
\text { (RA) }\end{array}$ & $\begin{array}{l}\text { Complejidad para abordar la relación y vínculo administrativo- } \\
\text { pedagógico con los apoderados. }\end{array}$ \\
\hline $\begin{array}{l}\text { Tareas administrativas } \\
\text { (TA) }\end{array}$ & $\begin{array}{l}\text { Evidencia la falta de conocimiento de las tareas administrativas que } \\
\text { implica el rol docente en el ejercicio profesional. }\end{array}$ \\
\hline $\begin{array}{l}\text { Experiencia en } \\
\text { prácticas (EP) }\end{array}$ & $\begin{array}{l}\text { Relevancia que entregan las experiencias en las prácticas para cada } \\
\text { etapa vivida en la formación docente. }\end{array}$ \\
\hline
\end{tabular}
van en la tabla 5.

En esta etapa del análisis, se generó una vinculación entre los códigos generados en el proceso de análisis de discurso (AD) y los conglomerados temáticos que emergieron desde el análisis de contenido (AC), con la finalidad de observar si ambos procesos de análisis tenían cercanía en la evidencia levantada, resultando que cada conglomerado pudo asociarse a alguno de os códigos existen- 
tes, esto bajo la significación que se les otorgó a los 11 conglomerados y los 15 códigos, existiendo un alto grado de coherencia capturándose así todos los conglomerados en los códigos, tal como puede observarse en la tabla 6 .

\begin{tabular}{|c|c|c|c|}
\hline Código & Conglomerado & Código & Conglomerado \\
\hline $\begin{array}{c}\text { Cambio } \\
\text { curricular (7) }\end{array}$ & 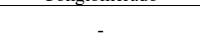 & Manejo de grupo (13) & $\begin{array}{c}\text { Manejo situaciones } \\
(112)\end{array}$ \\
\hline $\begin{array}{c}\text { Conflicto } \\
\text { escolar (6) }\end{array}$ & $\begin{array}{c}\text { Conflictos escolares } \\
(69)\end{array}$ & $\begin{array}{l}\text { Planificaciones } \\
(10)\end{array}$ & Planificar (26) \\
\hline $\begin{array}{c}\text { Escasa } \\
\text { implementación (9) }\end{array}$ & - & Proactividad (9) & $\begin{array}{l}\text { Gestión } \\
\text { clases (93) }\end{array}$ \\
\hline $\begin{array}{c}\text { Factores } \\
\text { externos (7) }\end{array}$ & - & $\begin{array}{l}\text { Problemas de } \\
\text { formación (16) }\end{array}$ & - \\
\hline $\begin{array}{c}\text { Fortaleza } \\
\text { disciplinar (9) }\end{array}$ & $\begin{array}{l}\text { Asignaturas } \\
\text { (54) }\end{array}$ & $\begin{array}{c}\text { Relación apoderados } \\
(9)\end{array}$ & $\begin{array}{c}\text { Relación } \\
\text { apoderados (32) }\end{array}$ \\
\hline $\begin{array}{l}\text { Fortaleza eje } \\
\text { prácticas }(9)\end{array}$ & $\begin{array}{c}\text { Prácticas } \\
\text { (53) }\end{array}$ & $\begin{array}{c}\text { Tareas } \\
\text { administrativas (11) }\end{array}$ & - \\
\hline $\begin{array}{l}\text { Fortaleza sistema } \\
\text { escolar (13) }\end{array}$ & $\begin{array}{c}\text { Contexto escolar } \\
\text { (109) }\end{array}$ & $\begin{array}{l}\text { Experiencia en } \\
\text { prácticas (18) }\end{array}$ & Experiencias (35) \\
\hline $\begin{array}{c}\text { Fortalezas } \\
\text { formación }(10)\end{array}$ & $\begin{array}{l}\text { Formación docente (78) } \\
\text { Rol Profesor (108) }\end{array}$ & & \\
\hline
\end{tabular}

Como cierre del análisis de discurso y a partir de los códigos conformados por las citas capturadas se generaron nuevos agrupamientos temáticos, los que a través del acercamiento semántico generaron las familias de códigos, que se presentan como la organización o agrupación de objetos o componentes principales lo que posibilita un trabajo más ordenado y ayuda a filtrar los elementos para una labor más enfocada (Muñoz \& Sagahún, 2017; Penalva, Alaminos, Francés \& Santacreu, 2015), lo que representa el cierre del proceso de análisis inductivo y propicia una significación más específica del fenómeno.

Lo anterior da como resultado la creación de 5 familias: (a) Familia Currículo, que da cuenta de las debilidades asociadas a los aspectos curriculares que integra el trabajo docente en el sistema escolar (currículum, planificaciones, gestión curricular, etc.), la que se conforma por los códigos Cambio curricular y Planificaciones; (b) Familia Debilidades de la Formación, las que se entienden como los nudos críticos que presentan los programas de formación en base a las reales demandas que implica el ejercicio profesional docente en la actualidad, la que se conforma por los códigos Manejo de grupo y Problemas de formación; (c) Familia Debilidades en el Sistema Escolar, que son las debilidades que presenta el docente y que dificultan su ejercicio profesional en el contexto escolar, la que se conforma por los códigos Tareas administrativas, Escasa implementación, Relación apoderados, Conflicto escolar y Factores externos; (d) Familia Fortalezas, que hace alusión a los aspectos favorables que tuvo la formación docente y que contribuyen a su desempeño óptimo como docente, la que se conforma por los códigos Fortalezas formación, Fortalezas sistema escolar, Fortaleza disciplinar y Fortaleza eje prácticas; y (e) Familia Prácticas, que vincula el valor de la experiencia vivida en las prácticas como acercamiento formal a la realidad escolar como futuro campo de desarrollo profesional docente, la que se conforma por los códigos Proactividad y Experiencia en prácticas.

En la «Familia Currículo» gran parte de los discursos aluden principalmente a los aspectos y condiciones actuales de los cambios curriculares, lo que ha dificultado que los docentes noveles puedan dar respuesta a los requerimientos en materia de currículum, lo anterior dado que su formación transitó en paralelo a estos cambios en educación, lo que no permitió generar ajustes en los planes de estudio durante su etapa de formación profesional.
«Reforzaría más en la universidad es el currículo, haría un ramo netamente que hable del currículo desde la página uno hasta la página ciento y tanto, que hable de las bases curriculares chilenas, cuáles son sus objetivos, que se los sepan casi de memoria los chicos al salir de la universidad» (Cód. CC. S7:18)

En efecto, gran parte de los cambios en educación a nivel de macro políticas ha tenido eco posterior a lo que se conoció como «Revolución Pingüina» (2006) y que tiene como hito político la derogación de la Ley Orgánica Constitucional de Educación (LOCE) el año 2009 y la instalación de la Ley General de Educación (LGE), con profundos cambios y transformaciones en educación, entre los cuales destaca la instalación de un nuevo currículum nacional llamado Bases Curriculares, como eje del trabajo técnico pedagógico de los docentes, impactando principalmente en el desarrollo de planificaciones de clase como ejercicio de programación de la enseñanza en los alumnos.

«Las herramientas que me entrega la universidad son básicamente en términos administrativos también son muy vagas porque esto se va actualizando constantemente, entonces uno se encuentra con que las planificaciones están primero en los planes y programas, ahora están las bases curriculares y esto va variando» (Cód. PL. S10:4)

$\mathrm{La}$ «Familia Debilidades de la Formación» se hace referencia a las tensiones de los diseños curriculares y su impacto en la formación docente, los cuales no han logrado responder eficazmente a las reales demandas que tiene hoy el trabajo en el sistema escolar.

«En la universidad trabajamos poco sobre temas importantes como convivencia, conflictos, etc. y yo siento que me falta mucho en esa área» (Cód. PF. S13:12)

Es así como muchas de las necesidades en este ámbito están vinculadas a problemas en la formación docente que en la actualidad se presentan como debilidades en los noveles y que dificultan su óptimo desempeño docente.

«Situaciones que se dan en el patio que a veces no se ven en el aula, son netamente situaciones de resolución de conflicto que tengan que ver con el compartir, con ver el fracaso como una posibilidad, una oportunidad de aprender la tolerancia a la frustración o la intolerancia a la frustración que tienen los niños»(Cód. MG. S11:1)

Para el caso de la «Familia Debilidades en el Sistema Escolar» los noveles establecen las principales debilidades que ellos presentan, las cuales se relacionan con tareas, acciones y toma de decisiones que a diario deben enfrentar y para lo cual no están lo suficientemente preparados, lo que dificulta su correcto desempeño profesional.

«El material que nos entregan en educación física es demasiado poco para los alumnos que tenemos, pero como nos han formado con muchos materiales, que de otra forma cuesta trabajar lo mejor posible» (Cód. EI. S6:14)

Estas dificultades muestran al novel sin las competencias docentes en estos ámbitos, lo que genera en él sensaciones de desconcierto, rabia, angustia y desmotivación, lo que sería a la larga una de las razones por las que podría desistir del ejercicio docente futuro.

«Es fundamental el tema de la convivencia escolar, yo creo que un ramo de convivencia escolar porque hoy en día nos estamos viendo inmersos en diversas situaciones, 
ya sea sociales, culturales entonces, el saber llevar a los chiquillos tener alguna estrategia o el tener alguna metodología en base a eso» (Cód. CE. S9:4)

En la «Familia Fortalezas» surgen respuestas favorables de los noveles en base a su formación docente, destacando que éstas son facilitadoras del desempeño de su labor y que con el tiempo afianzan su vocación, madurez y experiencia como profesores.

«Con el tiempo uno se va perfeccionando va aprendiendo de la experiencia de los problemas de los errores yo creo que con eso uno va de a poco dejando de ser novel pero lo que si uno nunca deja de aprender» (Cód. FSE. S9:7)

En este sentido los aspectos que favorecen el trabajo de los noveles tiene que ver con la relación que se dio en su formación entre lo aprendido y la utilidad práctica para desarrollar su labor, estableciendo coherencias de lo que trata para las universidades el formar docentes que respondan a las reales necesidades del sistema escolar.

«Se encuentra currículum de la educación, didáctica, debido a que estas asignaturas son de las que más se aproximan a la realidad escolar» (Cód. FD. S5:1)

«Se recibe una retroalimentación por parte de todos los entes que participan en el proceso de enseñanza - aprendizaje» (Cód. FF. S4:3)

La «Familia Prácticas» hace referencia al valor y significado que tiene la actividad del eje de prácticas como una experiencia previa de acercamiento progresivo del futuro docente, ya sea en su tramo inicial, intermedio o de cierre (profesional), entendiendo que esta actividad ofrece una experiencia similar a la realidad futura del ejercicio profesional docente, lo que vincula al estudiante en formación con el rol docente, simulando las tareas y deberes que en el futuro próximo tendrá con alguna institución educativa.

«Es una instancia de cometer errores para futuras acciones, como a su vez vivenciar situaciones y problemáticas del contexto» (Cód. EP. S3:2)

Otro elemento a destacar es que las prácticas estimulan la proactividad como respuesta a lo emergente de su quehacer, considerando permanentemente acciones como respuesta a esos requerimientos; en este sentido el futuro docente debe potenciar la rápida toma de decisiones para que lo propuesto tenga el impacto que espera.

«Ser profesor es, exactamente es una constante actualización y estar inserto constantemente, no dejarse estar, y esta es una labor que tiene que ver con la situación personal de cada uno» (Cód. PR. S10:6)

En relación a la experiencia en las prácticas se destacan las condiciones que se presentan en las prácticas para la comprensión detallada de lo que son los diferentes contextos escolares y con ello poder tener experiencias diversas que fortalezcan su rol docente. En este sentido, lo heterogéneo de los centros escolares hace que los futuros profesores convivan con realidades de todo tipo, no encasillándose en uno u otro tipo sino más bien convivir con experiencias de toda índole.

«Yo siento que lo mejor que me entrego la universidad es que yo pueda ser un profesor todo terreno que responda en todos los ámbitos ya sea en los sectores más bajos, en los sectores medios y en los sectores altos» (Cód. EP. S13:3)

\section{Discusión}

Hoy en día ser profesor requiere de personas que, durante su formación, hayan tenido diversas experiencias en favor del desarrollo de las competencias necesarias para responder a las tareas que implica su desempeño profesional, lo que en resumen configura un profesor que sea capaz de tener respuestas efectivas a cada una de las situaciones con que se enfrenta. Si bien esta consideración es clave para lo que será su desempeño profesional futuro, no puede ser una norma rígida para un profesor que comienza a vivenciar el complejo entramado de su profesión, más aun si en esta etapa el profesor novel carece de una experiencia y expertiz profesional, «constituyéndose en un momento clave en la trayectoria inicial de todo docente, siendo un periodo donde se realiza la transición de estudiante a profesor, que está marcado por tensiones y aprendizajes intensivos, el cual se desarrolla en contextos que generalmente resultan desconocidos para el docente principiante» (Carnevale, 2009), que para este caso ha sido breve y quizá no significativa.

Lo anterior demuestra que la formación docente no logra el desarrollo de todas las capacidades para desenvolverse a la par de quienes tienen mayor tiempo de ejercicio docente, lo que Gutiérrez et al. (2017) destaca como la funcionalidad de los aprendizajes mediante la contextualización y utilidad práctica en el contexto diario, más aun considerando que según Serra, Krichesky \& Merodo (2009) «el proceso para llegar a ser docente comienza con la propia experiencia escolar como alumno y continua durante toda la carrera profesional, identificándose las fases de: a) La experiencia como alumnos, b) La formación inicial como docentes, c) La iniciación de la vida profesional en las instituciones educativas, y d) La fase de formación permanente», lo que demuestra que la formación no termina con el tiempo de duración fijo sino más bien es un proceso que se extiende por mucho tiempo más en el ejercicio profesional.

En consideración, en esta investigación pudo conocer la percepción tienen los profesores noveles sobre su formación docente, lo anterior de acuerdo a la experiencia vivida en esta primera etapa de ejercicio profesional, evidenciándose aspectos que favorecen y desfavorecen su trabajo; los aspectos favorables (6) se centran en aspectos que tienen como principal foco la institución formadora (universidad), estos se orientan a aspectos externos vinculados a las prácticas (asignaturas de práctica, experiencias vividas, proactividad y sistema escolar), y por otra parte a aspectos internos como lo curricular y administrativo de la carrera (campo disciplinar y modelo de formación docente) lo que reviste una valiosa consideración debido a la importancia que los estudiantes tengan experiencias facilitadoras desde lo procedimental y también en lo administrativo, lo que se relaciona con Carreño et al. (2019) quien manifiesta que «el oficio del docente siempre es reconocido como proceso construido con experiencia cotidiana lo cual implica que más allá de los saberes, temas o técnicas que se consideren esenciales de la educación física, existiendo saberes que se inscriben en la relación cotidiana con las poblaciones, posiblemente relacionadas con habilidades afectivas, ociosas o culturales».

Dentro de los aspectos desfavorables (9) abordan en 
gran parte temas referidos nudos críticos que emergen desde el desempeño en el sistema escolar, y en los cuales la universidad no tiene responsabilidad directa sobre su manejo, transfiriéndole en cierta medida el deber de preparar, en base a las diferentes asignaturas, el enfrentar estas dificultades con herramientas efectivas para su resolución. Este componente, que en su mayoría obedece al funcionamiento de los centros escolares, transita entre los cambios que en la actualidad enfrenta en general la sociedad y replican en su particularidad las escuelas, como lo son los cambios curriculares en educación, los conflictos y el manejo de grupo, la implementación y recursos, aspectos técnico-pedagógicos y la relación con los apoderados.

Es evidente que, si bien los procesos de formación docente pueden estar determinados y organizados coherentemente, gran parte de las circunstancias que rodea el ejercicio profesional docente no son parte de los aprendizajes declarados en asignaturas o experiencias de las prácticas, lo que supone una formación descontextualizada de la realidad y que solo responde al campo de los saberes que de desprenden desde las disciplinas integradas en el currículum que conforma la proyección y transito formativo de un estudiante hacia su composición como futuro profesor, lo que Carreño et al. (2019) refuerza señalando que «la formación docente debe observarse como proceso trascendental de construcción de sujetos responsabilizados de la educación de otros sujetos, donde más allá de la regulación normativa, implica la constitución ética frente a las problemáticas de la educación en los contextos.

\section{Conclusiones}

La formación del profesorado está en el foco de la contingencia educacional por el valor que tiene para el sistema educativo la calidad de sus docentes, más aun cuando Barber $\&$ Mourshed (2008) en el informe McKinsey manifiestan que la calidad de un sistema educativo se basa en la calidad de sus docentes. Este escenario configura la necesidad de contar con profesores que den respuesta oportuna y efectiva a cada una de las situaciones que emergen en la escuela, lo que implica un alto estándar de desempeño profesional docente.

Es así como la evidencia encontrada da cuenta que los docentes en sus primeros años de trabajo no tienen dominio acabado de las acciones que desarrollan, existiendo bastantes dificultades o nudos críticos que datan desde la formación docente como también son parte del poco bagaje de experiencia que tienen como nuevos docentes. Este escenario se configura en función de una serie de circunstancias positivas o negativas que surgen en este tiempo, manifestando que en el caso de las negativas no facilitan su desempeño por no tener respuestas a los requerimientos que ellas implican, entendiendo que las positivas se configuran como un dispositivo que fortalece su rol y acción docente, lo que hace necesario que los docentes noveles al momento de integrarse a las instituciones educativas tengan una red de apoyo que le faciliten su inicio profesional y de esa forma fidelizar la vocación, sus competencias y el desempeño docente, impactando positivamente esta etapa del novel.

En cuanto a las principales dificultades con que se en- frentan los novatos al iniciarse como docentes, estas dan cuenta de aspectos del ámbito técnico-pedagógicos (tareas administrativas y planificaciones), en el ámbito de la convivencia escolar (conflictos, relación con apoderados y manejo de grupo) y en el ámbito de las condiciones para el desarrollo de clases (escasa implementación, tecnología y motivación estudiantes), lo que en resumidas cuentas muestra que la principal dificultad del docente nuevo se configura principalmente por su falta de experiencia, entendiendo que su formación integra experiencias en estos ámbitos, las que al parecer no suelen ser significativas según la evidencia encontrada. He aquí un punto clave en lo que respecta al cumplimiento de expectativas del novel en sus primeros años, puesto que las dificultades y problemas que no logra resolver le genera desmotivación e inclusive propicia altas probabilidades de abandono de la profesión en el corto plazo.

En relación al proceso de formación docente es importante señalar que la evidencia muestra que las principales fortalezas que presenta se sitúan en el acabado conocimiento disciplinar de la profesión, lo valioso que fue el eje de las prácticas como oportunidad de conexión con la realidad y de aportación de experiencias docentes en la escuela, y la instalación profunda del rol docente en los estudiantes, que fortalecen al nuevo profesor con competencias de base para iniciar su trayectoria profesional, manifestando que la principal responsabilidad que tienen las instituciones de educación superior es ofrecer oportunidades que impacten las dimensiones que conforman al estudiante, entendiendo que estas experiencias deben estar necesariamente contextualizadas con la realidad futura a la cual se verá enfrentado el profesor en formación, asegurando con esto que toda actividad curricular y formativa tendrá un aprendizaje significativo, lo que permitirá la apropiación efectiva de las competencias para su trabajo en el tiempo.

Si bien las prácticas fueron evidencia de los aspectos positivos que manifestaron a través de la evidencia, es importante resaltar que en todos los casos la vivencia generada por los procesos de prácticas durante el transcurso de la formación docente logro acentuar en los noveles el sentido práctico del quehacer del profesor, acercándolo de manera casi real a las distintas tareas que cumple un docente en ejercicio, siendo el eje de prácticas un punto que articula los saberes teóricos y prácticos en el trabajo in situ con alumnos en el sistema escolar. En este sentido, la práctica es una actividad curricular clave en lo que a formar un profesional respecta, lo anterior en virtud de que, si bien la práctica tiene una funcionalidad limitada para el estudiante en razón que la responsabilidad última la tiene el profesor titular de la clase, le permite al futuro docente vivir la experiencia de programar enseñanza, relacionarse con alumnos, regular la convivencia en la clase, evaluar logros de aprendizaje y por sobre todo posicionarse gradualmente en el rol docente.

En síntesis, la formación de profesores debe considerar experiencias relevantes que aseguren el desarrollo de las competencias para desempeñarse correctamente, poniendo especial atención a las diversas dificultades que enfrentan los profesores noveles en sus primeros años de ejercicio profesional, lo anterior para generar los ajustes correspondientes a las actividades curriculares contextualizadas de acuerdo a la realidad futura del docente, entendiendo que es 
imposible vivir el rol de un profesor sin serlo, pero que exista un acercamiento progresivo en base a actividades como las prácticas o didácticas de simulación de las asignaturas que componen los planes de estudio.

\section{Referencias}

Alliaud, A. (2004). La experiencia escolar de maestros inexpertos. Biografías, trayectorias y práctica profesional. Revista Iberoamericana de Educación. 34(1), 1-11. Recuperado de: https://doi.org/https://doi.org/10.35362/ rie3412888.

Almonacid-Fierro, A., Merellano-Navarro, E., Feu, S., Vizuete, M. \& Orellana, R. (2019). Perspectiva cualitativa en la construcción del conocimiento didáctico del contenido del profesorado de Educación Física. Retos, 36(2), 459-468.

Álvarez,A. (2007). Investigación Cualitativa. Editorial Ciencias Médicas. La Habana. Cuba.

Artiles, L. (1990). Análisis de discurso: Introducción a su teoría y práctica. Editado por centro Cultural Poveda. Santo Domingo. República Dominicana.

Ávalos, B. (2002). Profesores para Chile: Historia de un Proyecto. Santiago: Ministerio de Educación.

Ávalos, B. (2003). La formación docente inicial en Chile. Digital Observatory for Higher Education in Latin America and the Caribbean. Unesco Iesalc. Recuperado de: https:/ /www.oei.es/historico/docentes/info_pais/ informe formacion docente chile iesalc.pdf.

Ávalos, B. (2009). La inserción profesional de los docentes. Revista de Currículum y Formación de profesorado. Volumen $13, \mathrm{~N}^{\circ} 1,43-59$.

Barber, M. \& Mourshed, M. (2008). Cómo hicieron los sistemas educativos con mejor desempeño del mundo para alcanzar sus objetivos. Programa de Promoción de la Reforma Educativa en América Latina y el Caribe (PREAL). Primera edición. Santiago de Chile. Recuperado de: www.preal.org/publicacion.asp

Barrera, M. \& Hinojosa, C. (2017). Incidencia del proceso de la práctica profesional en las dimensiones de la formación docente de estudiantes de pedagogía en educación física. Revista Pensamiento Educativo. 54(2), 1-15.

Boerr, I. (2014). El largo camino de convertirse en profesor. Revista Docencia del Colegio de Profesores de Chile. Año XIX. N 54. 52-63. Santiago de Chile. Recuperado de: http://revistadocencia.cl/web/index.php/edicionesanteriores/60-docencia54

Bores, D., Marín, A., Méndez, C., Mijarra, J.J. \& Delfa, J. (2020). Aspectos metodológicos clave para la formación de futuros maestros de Educación Física. Retos, 37, 572-578.

Briones, G. (1999). Filosofía y teoría de las Ciencias Sociales: dilemas y propuestas para su construcción. Domen Ediciones. Santiago de Chile.

Calvo, G., \& Camargo, M. (2015). Hacer escuela en la formación de docentes nóveles. Páginas De Educación, 8(1), 73-91. Recuperado de https://doi.org/10.22235/pe.v8i1.496

Cañadas, L., Santos-Pastor, M. \& Castejón, F. (2019). Competencias docentes en la formación inicial del profesorado de educación física. Retos, 2041(35), 284-288.
Carnevale, S. (2009). Las dificultades de los profesores noveles para la enseñanza de la Historia. XII Jornadas Interescuelas / Departamento de Historia, Facultad de Humanidades y Centro Regional Universitario Bariloche. Universidad Nacional del Comahue, San Carlos de Bariloche. Recuperado de: http://cdsa.aacademica.org/ 000-008/859

Carreiro Da Costa, F., Carvalha, L., Diniz, J. \& Pestana, C. (1996). As expectativas de exercício profíssíonal dos alunos de um curso que habilita para a docencia: A formacáo (nao) passa por aqui? Forrnacáo de Professores em Educacáo Física. Concepcóes, Investigacáo, Prática (pp. 57-74). Lisboa: Edicóes FMH. Carreño, J., Velasco, A., Domínguez, S. \& Martín, J. (2019). ¿Qué se investiga en formación docente en educación física y recreación? Retos, 2041(36), 3-8.

Cazau, P. (2006). Introducción a la investigación en Ciencias Sociales. Rundi Nuskín Editor. Tercera edición. Buenos Aires. Argentina.

Corbetta, P. (2007). Metodología y técnicas de investigación social. Edición revisada. Editorial Mc Graw-Hill. México.

Cornejo, J. (1999). Profesores que se inician en la docencia: algunas reflexiones al respecto desde América Latina. Revista Iberoamericana De Educación, 19, 51-100. Recuperado de: https://doi.org/https://doi.org/10.35362/ rie1901055

CPEIP (2014). Estándares orientadores para carreras de Pedagogía en Educación Física. Centro de Perfeccionamiento, Experimentación e Investigaciones Pedagógicas del Ministerio de Educación (Mineduc). Santiago de Chile. Recuperado de: https://www.cpeip.cl/wp-content/ uploads/2018/09/Est\%C3\%A1ndares_Educacion_fisica2.pdf

Delgado, J. \& Gutiérrez, G. (1995). Métodos y Técnicas Cualitativas de Investigación en Ciencias Sociales. Editorial Síntesis. Madrid. España

Denzin, N. \& Lincoln, Y. (Coords.). (2015). Métodos de recolección y análisis de datos. Manual de investigación Cualitativa. Volumen IV. Primera Edición. Editorial Gedisa. Barcelona. España.

Díaz del Cueto, M. \& Aguado, R. (2012). Percepción de competencia del profesorado de educación física con experiencia sobre la tarea como recurso didáctico. Retos, 22, 16-18.

Dooley, R. \& Levinsohn, S. (2007). Análisis del discurso: Manual de conceptos básicos. Instituto Lingüístico de verano. Lima. Perú.

Fernández, J.M. (2005). La auto-evaluación como práctica de la democracia y la dignidad. In Fernández, J.M. \& Sicilia, A. (Eds.). La otra cara de la enseñanza: la educación física desde una perspectiva crítica. Barcelona. España.

Flick, U. (2007). Introducción a la investigación cualitativa. Segunda Edición. Ediciones Morata. España.

García, C.M. (2006). Políticas de inserción a la docencia de eslabón perdido a puente para el desarrollo profesional docente. Editado por PREAL, GTD y la Universidad de Bogotá Jorge Tadeo Lozano. Bogotá, Colombia. Recuperado de: http://www.ub.edu/obipd/docs/ politicas_de_insercion_a la docencia_del_eslabon_perdido_al_puen 
te para el desarrollo profesional docente garcia c m.pdf Gimeno Sacristán, J. \& Pérez Gómez, A. (2008). Comprender y transformar la enseñanza. Editorial Morata. España.

Gold, Y. (1996). Beginning teacher support. Attrition, mentoring, and induction. In C. B.Courtney (Ed.) Review of Research in Education, 16, pp.548-594. Washington, DC:American Educational Research Association.

Grawitz, M. (1984). Métodos y técnicas de las ciencias sociales. Biblioteca de Ciencias Sociales. Editia Mexicana. México.

Gutiérrez-Díaz, D., García-López, L.M., Pastor-Vicedo, J.C., Romo-Pérez, V., Eirín-Nemiña, R. \& Fernández-Bustos, G. (2017). Percepción del profesorado sobre la contribución, dificultades e importancia de la Educación Física en el enfoque por competencias. Retos, 2041(31), 34-39.

Hernández, R., Fernández, C. \& Baptista, P. (2014). Metodología de la Investigación. Sexta edición. Editorial McGraw-Hill. México.

Imbernón, F. (1997). La formación y el desarrollo profesional del profesorado. Hacia una nueva cultura profesional. Editorial GRAO. Barcelona, España.

Iñiguez, L. (ed.) (2011). Análisis de discurso: Manual para las ciencias sociales. Editorial UOC. Barcelona, España.

Izcara, S. (2014). Manual de Investigación Cualitativa. Primera edición. Editorial Fontamara. México.

Keats, D. (2009). Entrevista: Guía práctica para estudiantes y profesionales. Primera edición. Editorial Mc GrawHill. México.

López, V., Pérez, D., Manrique, J. \& Monjas, R. (2016). Los retos de la Educación Física en el siglo XXI. Retos, 29, 182-187.

Núñez, I. (2004). La identidad de los docentes. Una mirada histórica en Chile. Presentada al XIV Congreso Mundial de Ciencias de la Educación. Santiago de Chile. Asociación Mundial de Ciencias de la Educación (AMCE) y P. Universidad Católica de Chile.

Ortiz, A. (2015). Enfoques y métodos de investigación en las ciencias sociales y humanas. Ediciones de la U. Bogotá. Colombia.

Paez, J. \& Hurtado, J. (2019). Formación inicial docente en profesores de educación física. Levantamiento de competencias específicas a partir de las necesidades del medio educativo. Retos, 2041(35), 61-66.

Patton, M. (1990). Qualitative Evaluation and Research
Methods. Second Edition. London: Sage Publications.

Penalva, C., Alaminos, A., Francés, F. \& Santacreu, O. (2015). La investigación cualitativa: técnicas de investigación y análisis con Atlas.Ti. PYDLOS Ediciones. Universidad de Cuenca. Ecuador.

Pérez, G. (2002). Investigación cualitativa: Retos e interrogantes. Técnicas y análisis de datos, segunda parte. $3^{\mathrm{a}}$ edición. Editorial la Muralla S.A. Madrid: España.

Rodríguez, I., Del Valle, S. \& De la Vega, R. (2018). Revisión nacional e internacional de las competencias profesionales de los docentes de Educación Física. Retos, 34, 383-388.

Ruiz, J. (2012). Metodología de la investigación cualitativa. $5^{\text {a }}$ edición. Editorial Universidad de Deusto. España.

Strauss, A. \& Corbin, J. (2002). Bases de la investigación cualitativa. Técnicas y procedimientos para desarrollar la teoría fundamentada. Primera edición (en español). Editorial Universidad de Antioquia. Colombia.

Strauss, A. (1987). Qualitative analysis for social scientists. Cambridge University Press. New York, EEUU.

Trigueros, C., Rivera, E. \& Rivera, I. (2018). Técnicas conversacionales y narrativas Investigación Cualitativa con Software NVivo. Editan: Universidad de Granada \& Escuela Andaluza de Salud Pública. España.

Valles, M. (2002). Entrevistas cualitativas. Centro de investigaciones sociológicas. Cuadernos metodológicos №32. Primera edición. Madrid. España.

Van Maanen, J. (2011). Tales of the field: On writing ethnography. Second edition. University of Chicago Press. EEUU.

Vasilachis de Gialdino, I. (Coord.). (1996). Estrategias de investigación cualitativa. Primera edición. Editorial Gedisa S.A. Barcelona. España

Veenman, S. (1984). Perceived problems of beginning teachers. Review of Educational Research, vol. 54, $\mathrm{n}^{\circ} 2$, pp. 143-174.

Vilca, E. (2005). El profesor novel. Chile: Educrea. Recuperado de: https://educrea.cl/el-profesor-novel.

Vonk, J. (1996). A knowledge base for mentors of beginning teachers: result of a Dutch experience. In Mcbrigde, R. Theacher Education Policy: Some issues areising from research and practice. Falmer Press. London and Washington, pp.113-134.
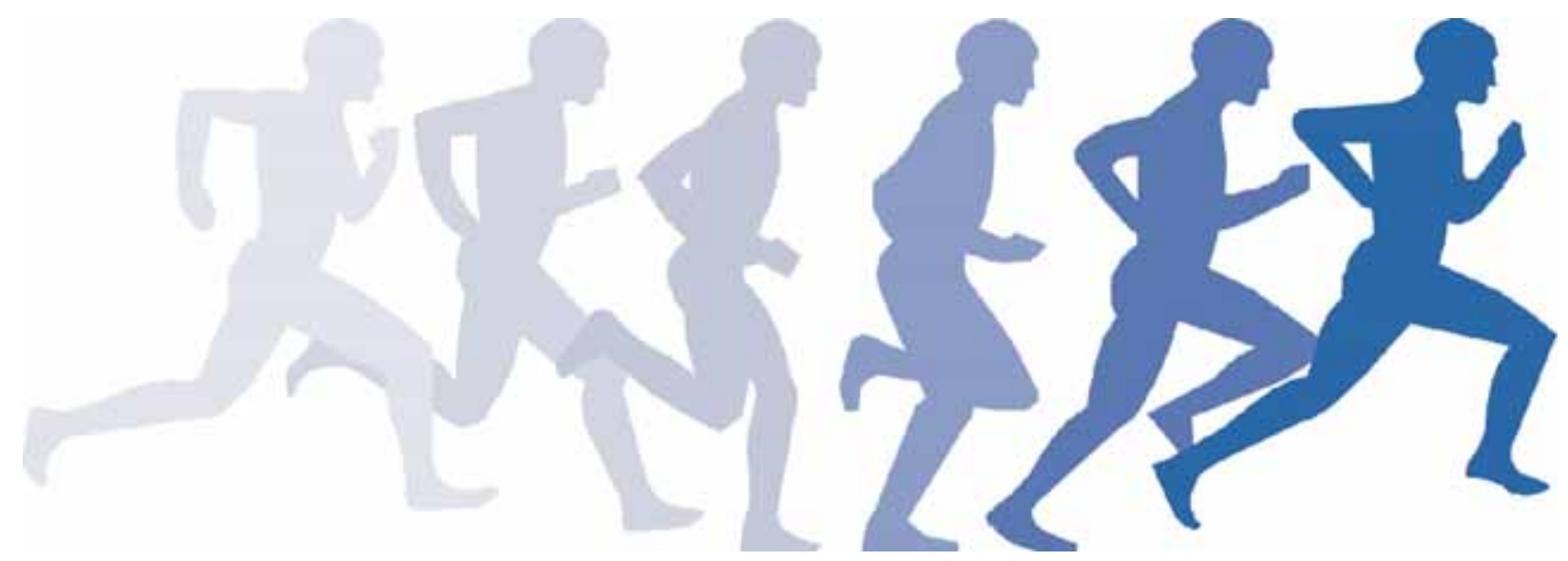